\title{
Extending coprime sensor arrays to achieve the peak side lobe height of a full uniform linear array
}

\author{
Kaushallya Adhikari ${ }^{*}$, John R Buck and Kathleen E Wage ${ }^{2}$
}

\begin{abstract}
A coprime sensor array (CSA) is a non-uniform linear array obtained by interleaving two uniform linear arrays (ULAs) that are undersampled by coprime factors. A CSA provides the resolution of a fully populated ULA of the same aperture using fewer sensors. However, the peak side lobe level in a CSA is higher than the peak side lobe of the equivalent full ULA with the same resolution. Adding more sensors to a CSA can reduce its peak side lobe level. This paper derives analytical expressions for the number of extra sensors to be added to a CSA to guarantee that the CSA peak side lobe height is less than that of the full ULA with the same aperture. The analytical expressions are derived and compared for the uniform, Hann, Hamming, and Dolph-Chebyshev shadings.
\end{abstract}

Keywords: Array tapers; Coprime sensor array; Dolph-Chebyshev; Hamming; Hann; Non-uniform linear arrays; Shading

\section{Introduction}

A sensor array spatially samples a propagating space-time signal. A beamformer combines the samples measured by an array to estimate properties of the space-time signal, such as the direction of arrival for signals of interest [1]. The beam pattern associated with a beamformer shows the gain for an input plane wave with a given wavenumber and temporal frequency. The beam pattern plays a role analogous to the frequency response in time domain processing. The main lobe width and side lobe heights are the two important beam pattern characteristics. Since the main lobe width in a beam pattern is inversely proportional to the array's ability to resolve signals arriving from different directions, a narrow main lobe is desirable. Since the peak side lobe height in a beam pattern is inversely proportional to the beamformer's ability to reject noise and interference and detect weak signals, a small peak side lobe height is desirable. A larger array aperture means a narrower main lobe width, hence better resolution. However, for a fixed intersensor spacing, greater

\footnotetext{
*Correspondence: kaushallyaadhikari@gmail.com

1 Electrical and Computer Engineering Department, University of

Massachusetts Dartmouth, 285 Old Westport Road, North Dartmouth, MA 02747, USA

Full list of author information is available at the end of the article
}

aperture requires more sensors thus increasing the installation and maintenance cost of the array. The intersensor spacing determines whether there are grating lobes in the beam pattern. If the intersensor spacing is more than half of the wavelength of the narrowband signal to be sampled, there are grating lobes meaning that the sampled signals are spatially aliased. The arrangement of sensors in the array, the weights provided to different sensors, the intersensor spacing, and the number of sensors determine the beamformer's performance. The array geometry sets certain restrictions on the beamformer. For example, a linear array cannot resolve more than one angular component of the signal's direction of arrival [1].

A beam pattern characterizes the response for a specific set of weights. In practice, observed data will be processed with a set of weight vectors, designed for the bearings of interest to estimate the spatial power spectrum. The spatial power spectrum is also known as the scanned response for the data. The scanned response is the spatial analogue of the power spectrum of a time series in time domain processing. The different beam patterns used to compute the scanned response for different look directions are commonly modulated or shifted versions of a common prototype beam pattern. Thus, it is important

\section{Springer}

(c) 2014 Adhikari et al: licensee Springer. This is an Open Access article distributed under the terms of the Creative Commons Attribution License (http://creativecommons.org/licenses/by/4.0), which permits unrestricted use, distribution, and reproduction in any medium, provided the original work is properly credited. 
to design a weight vector that has a beam pattern with the desired main lobe width and side lobe height.

The array shading (or tapering) that multiplies each sensor's measurement establishes trade-offs between the main lobe width and the side lobe heights. In general, shadings that result in narrower main lobes (better resolution) have higher peak side lobes (poorer ability to detect weak signals) and vice versa. This paper focuses on coprime linear arrays for different shadings. A coprime sensor array (CSA) is a non-uniform array created by interleaving two undersampled uniform linear arrays where the undersampling factors have no common factors greater than one $[2,3]$. This paper compares the performance of CSAs with the performance of uniform linear arrays (ULAs) with half wavelength sensor spacing, the same shadings, and comparable aperture.

Several studies have suggested that non-uniform linear arrays may outperform a ULA in terms of number of sensors, resolution, or side lobe attenuation [4-11]. Most non-uniform linear array designs have more than a half wavelength average intersensor spacing. Hence, for a given number of sensors, these non-uniform linear arrays can achieve larger aperture and thereby higher resolution than a standard ULA. For a given resolution, these nonuniform linear arrays require fewer sensors. Therefore, these non-uniform linear arrays are more efficient than their uniform counterparts. However, none of the methods in [4-11] offer a closed form expression for the sensor locations. The consistent interelement spacing of ULAs make these arrays significantly simpler to assemble and maintain than the highly variable interelement spacings required in many non-uniform linear arrays. The novel technique proposed by Pal and Vaidyanathan in references $[12,13]$ nests two or more ULAs systematically to create a non-uniform linear array that can detect $\mathcal{O}\left(N^{2}\right)$ sources using only $\mathcal{O}(N)$ sensors. However, the sensors can be very close to each other and there can be a problem of mutual coupling.

More recently, Vaidyanathan and $\mathrm{Pal}[2,3]$ proposed another type of non-uniform linear array called a CSA. This array design technique takes advantage of two integers being coprime (having greatest common divisor 1). A CSA can provide the resolution of a fully populated ULA with $\mathcal{O}(N)$ sensors using just $\mathcal{O}(\sqrt{N})$ sensors. For large $N, \mathcal{O}(\sqrt{N}) \ll \mathcal{O}(N)$. Thus, a CSA requires far fewer sensors than a full ULA, substantially reducing the installation, maintenance, and processing costs in the system. Vaidyanathan and Pal's array design technique provides a simple closed form expression for sensor locations. The elements of the CSA are several wavelengths apart on average, although a few sensor pairs are only a half wavelength apart. Hence, this technique reduces mutual coupling between the elements of the array. Another advantage of this CSA structure is that the
CSA is formed by combining two ULAs. The two ULAs are hereafter called subarrays. These uniform linear subarrays are straightforward to assemble as noted above. However, the CSA peak side lobe is higher than the peak side lobe of a ULA with the equivalent resolution. The side lobe height of a full ULA is insensitive to the total aperture, but the side lobe height of the CSA beam pattern may be decreased by increasing the aperture, as we discuss in more detail below. Consequently, the CSA peak side lobe can be made equal to the ULA peak side lobe by adding more sensors to each subarray [2,3]. We will refer to these CSAs with additional sensors as extended CSAs (ECSAs). This paper derives the number of additional sensors required in the subarrays for a CSA to match the peak side lobe of a ULA with the equivalent resolution for different standard tapers (uniform, Hann, and Hamming). Additionally, for the Dolph-Chebyshev taper, this paper derives the number of additional sensors required in the subarrays to achieve the peak side lobe of a ULA without matching the resolution.

Section 2 discusses CSAs and ECSAs in detail. Section 3 derives the analytical expressions for the total number of sensors in the ECSAs for different shadings: uniform, Hann, Hamming, and Dolph-Chebyshev. Section 4 provides some examples of the ECSA beampatterns. Section 5 compares different types of shadings for an ECSA in terms of the total number of sensors.

\section{Coprime sensor arrays}

A CSA consists of two undersampled ULAs with $M$ and $N$ elements respectively, where $M$ and $N$ are coprime integers $[2,3]$. The undersampled ULAs are called subarrays, and they have $N \lambda / 2$ and $M \lambda / 2$ intersensor spacings, where $\lambda$ is the wavelength of the narrowband signal to be sampled. The CSA is formed by interleaving the two subarrays. The sensor at location 0 is shared by both subarrays. This paper will refer to the subarray with $M$ sensors and intersensor spacing $N \lambda / 2$ as subarray 1 and the subarray with $N$ sensors and intersensor spacing $M \lambda / 2$ as subarray 2 . Figure 1a depicts two subarrays with coprime pair $(4,5)$. Figure $1 \mathrm{~b}$ shows how the CSA interleaves the two subarrays to obtain a non-uniform linear array. The total number of sensors in the CSA is $M+N-1$. However, the CSA aperture is $M(N-1) \lambda / 2$ (assuming $N>M$ ). Hence, the CSA resolution is comparable to a fully populated ULA with $M N$ sensors spaced every $\lambda / 2$, which will be called a full ULA in the rest of the paper.

To design a CSA that has the resolution of an $L$ element ULA, we need to choose the coprime integers $M$ and $N$ such that $M \cdot N=L$. There can be many possible choices for the coprime pair $(M, N)$ for a given $L$. For example, for $L=30$, we have three choices for a coprime pair: $(2,15),(3,10)$, and $(5,6)$. However, for a given $L$, the 


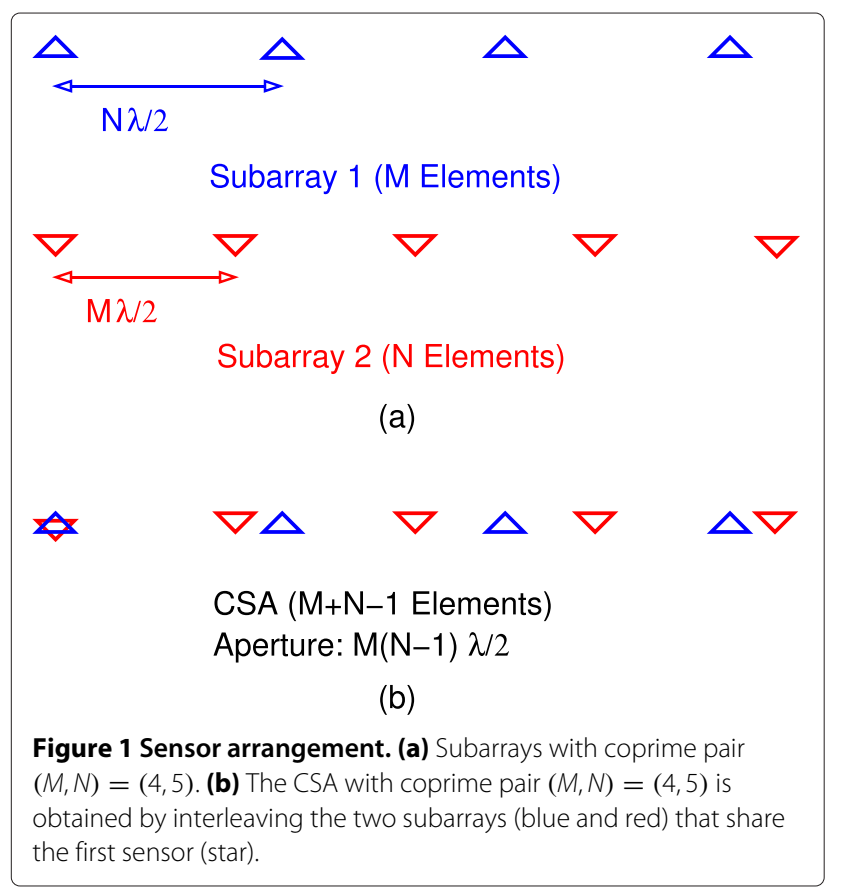

optimal coprime pair in terms of total number of sensors can be derived by solving the optimization problem:

$$
\begin{aligned}
\text { Minimize } & M+N \\
\text { subject to } & M N=L \\
& M>0 \\
& N>0
\end{aligned}
$$

The solution to the optimization problem is $M=N=$ $\sqrt{L}$, which is not a valid solution because $M$ and $N$ have to be coprime. As we demonstrated in [14], the valid optimal coprime pair is the one that has $M$ and $N$ as close as possible. For $L=30$, the coprime pair $(5,6)$ is optimal in minimizing the total number of sensors. The rest of the paper assumes that the CSA uses a coprime pair optimizing the number of sensors such that $N=M+1$.

For a standard linear array, a beam pattern $B(u)$ shows the gain of an array with given weights to a plane wave signal as a function of the direction of arrival $u=\cos \theta$, where $\theta$ is the angle with respect to array axis [1]. The effect of the beam pattern on the spatial power spectrum is given by converting to $\mathrm{dB}$ with $20 \log _{10}|B(u)|$, implicitly squaring the beam pattern to give units of power. When the sensors in an array are all equally weighted, the array is described as rectangular or uniformly shaded and has a relatively high side lobe peak of $-13 \mathrm{~dB}$ relative to the main lobe. These rectangularly shaded ULAs set the practical upper limit on side lobe height.

The CSA beamformer operates slightly differently, multiplying one subarray output with the complex conjugate of the second subarray output $[2,3]$. The resulting product of subarray beam patterns directly computes the spatial power spectrum response to plane waves for a set of weights applied. Figure 2 shows the steps in beamforming in a CSA. Here and in the sequel, we will refer to the beam pattern of the subarray beamformer as the subarray beam pattern. The subarray beam patterns $B_{1}(u)$ and $B_{2}(u)$ are given by

$$
\begin{aligned}
& B_{1}(u)=\frac{1}{M} \frac{\sin \left(\frac{\pi M N}{2} u\right)}{\sin \left(\frac{\pi N}{2} u\right)}, \\
& B_{2}(u)=\frac{1}{N} \frac{\sin \left(\frac{\pi M N}{2} u\right)}{\sin \left(\frac{\pi M}{2} u\right)} .
\end{aligned}
$$

Figure 3 shows the individual subarray beam patterns for the arrays in Figure 1, as well as those for the CSA and the full ULA. The product processing of the CSA beamformer leads to the spatial power spectrum $10 \log _{10}\left|B_{1}(u) \cdot B_{2}^{*}(u)\right|=10 \log _{10}\left|B_{1}(u)\right|+10 \log _{10}$ $\left|B_{2}(u)\right|$. To reflect the contribution of each subarray to the total CSA spatial power spectrum estimate, Figure 3 plots the subarray beam patterns in $10 \log _{10}(\cdot)$ scale. Since the sensors in the subarrays are more than a half-wavelength apart, the subarray beam patterns have grating lobes. The grating lobes of the first subarray are at the integer multiples of $2 / N$, and the grating lobes of the second subarray are at the integer multiples of $2 / M$. Figure 3 is drawn for $M=4$ and $N=5$, but the important locations of the grating lobes and widths have been identified in terms of the general expressions. Because $M$ and $N$ are coprime, the grating lobes from the two subarrays are at different locations. Taking the product of one beam pattern with the complex conjugate of the other generates the product beam pattern that has no grating lobes and the main lobe width is $4 /(M N)$, which is equal to the main lobe width of a full ULA with $M N$ sensors. Hence, a CSA with $M+N-1$ elements offers the resolution of an $M N$ element full ULA.

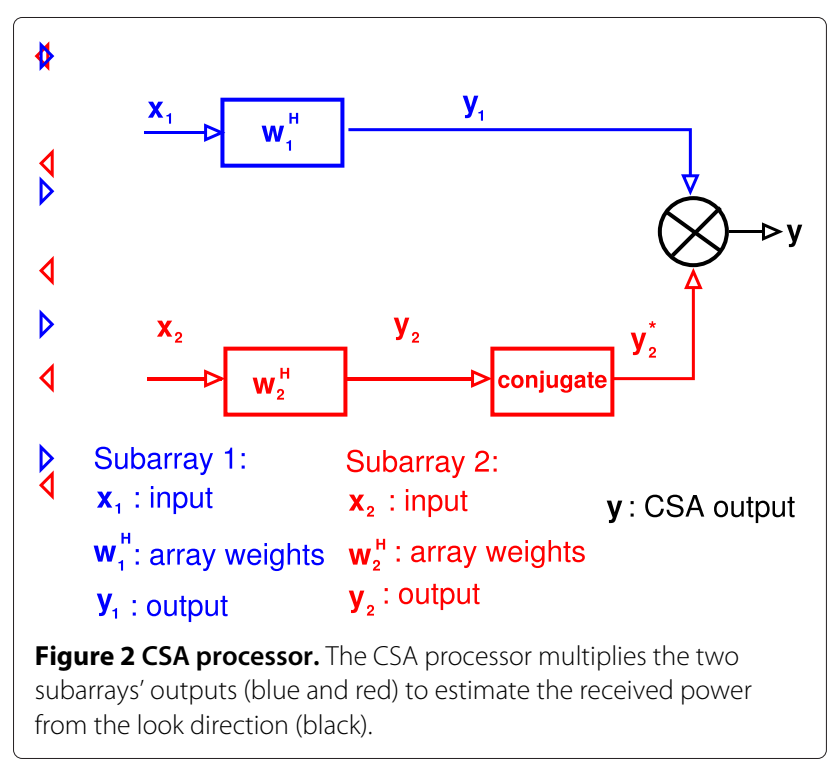



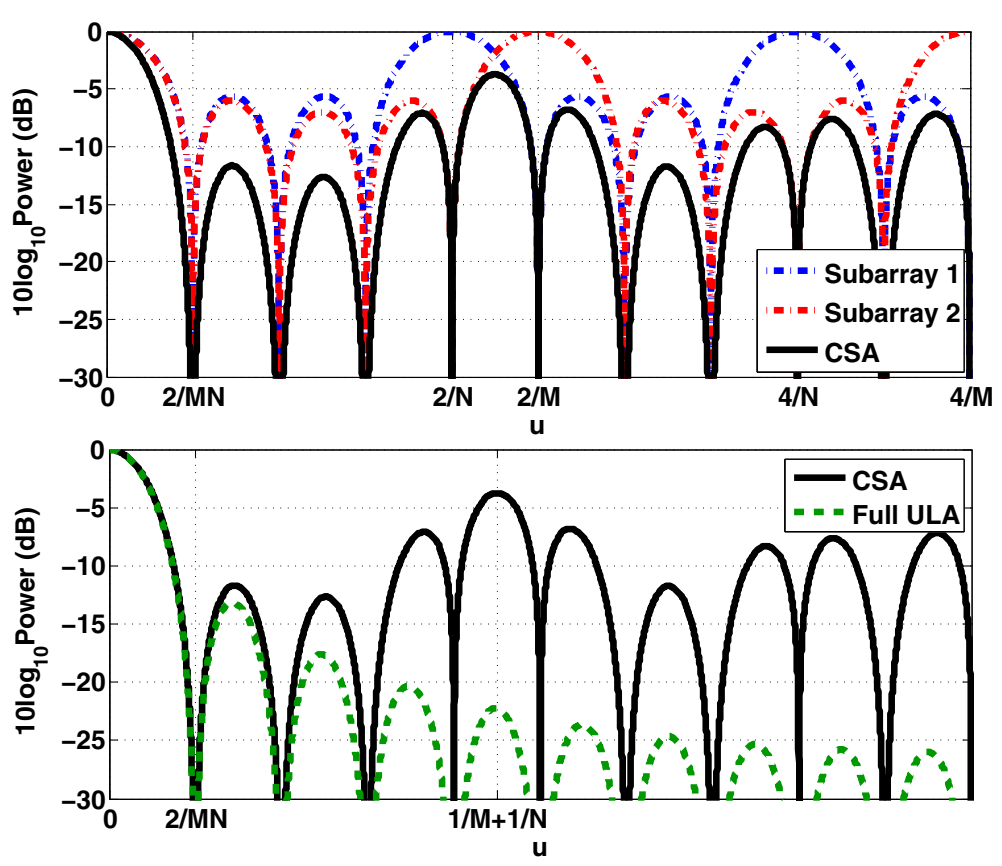

Figure 3 Beam patterns of the subarrays with four and five sensors, the CSA and the full ULA. The blue dashed-dot line is subarray 1 beam pattern in $10 \log _{10}(\cdot)$ scale. The red dashed-dot line is subarray 2 beam pattern in $10 \log _{10}(\cdot)$ scale. The black solid line is the CSA output power spectral density. The green dashed line is the equivalent full ULA's beam pattern in dB.

Figure 3 also demonstrates that the CSA peak side lobe is greater than the full ULA peak side lobe. Vaidyanathan and Pal suggest adding more sensors to each subarray to reduce the peak side lobe level in the CSA [2,3]. Adding more sensors to each subarray while keeping the intersensor spacing fixed reduces the main lobe and grating lobe widths of the subarrays. This reduction of the main lobe and grating lobe widths decreases the peak side lobe of the CSA beam pattern formed by the product of the two overlapping grating lobes in the subarray beam patterns, such as near $u=1 / M+1 / N=0.45$ in the top panel of Figure 3 . Vaidyanathan and Pal do not give explicit guidelines to determine the number of sensors to be added to the subarrays nor do they explain how changing the subarray shadings will impact the number of sensors required.

To understand the effect of high CSA peak side lobe, consider the detection of two sources using uniformly weighted CSA processor. The input to the first subarray is

$$
\mathbf{x}_{1}=A_{s} \mathbf{v}_{1 s}+A_{i} \mathbf{v}_{1 i}
$$

where $A_{s}$ and $A_{i}$ are the complex input signal amplitudes, $\mathbf{v}_{1 s}$ and $\mathbf{v}_{1 i}$ are the $M$ element direction vectors corresponding to the first subarray for directions $u_{s}$ and $u_{i}$. The $n$th elements of the direction vectors $\mathbf{v}_{1 s}$ and $\mathbf{v}_{1 i}$ are $\exp \left(j \pi \cdot u_{s} \cdot N \cdot(n-1)\right)$ and $\exp \left(j \pi \cdot u_{i} \cdot N \cdot(n-1)\right)$, respectively. Similarly, the input to the second subarray is

$$
\mathbf{x}_{2}=A_{s} \mathbf{v}_{2 s}+A_{i} \mathbf{v}_{2 i}
$$

where $\mathbf{v}_{2 s}$ and $\mathbf{v}_{2 i}$ are the $N$ element direction vectors corresponding to the second subarray for directions $u_{s}$ and $u_{i}$. The $n$th elements of the direction vectors $\mathbf{v}_{2 s}$ and $\mathbf{v}_{2 i}$ are $\exp \left(j \pi \cdot u_{s} \cdot M \cdot(n-1)\right)$ and $\exp \left(j \pi \cdot u_{i} \cdot M \cdot(n-1)\right)$, respectively. When the subarrays are steered to look at direction $u_{L}$, the weight vectors for the two subarrays are $\mathbf{w}_{1 L}=\mathbf{v}_{1 L} / M$ and $\mathbf{w}_{2 L}=\mathbf{v}_{2 L} / N$, respectively, where $\mathbf{v}_{1 L}$ and $\mathbf{v}_{2 L}$ are the $M$ and $N$ element direction vectors for the two subarrays. The outputs of the two subarrays when steered to direction $u_{L}$ are

$$
y_{1 L}=A_{s} \mathbf{v}_{1 L}^{H} \mathbf{v}_{1 s} / M+A_{i} \mathbf{v}_{1 L}^{H} \mathbf{v}_{1 i} / M
$$

and

$$
y_{2 L}=A_{s} \mathbf{v}_{2 L}^{H} \mathbf{v}_{2 s} / N+A_{i} \mathbf{v}_{2 L}^{H} \mathbf{v}_{2 i} / N,
$$

The resulting CSA spatial power spectrum estimate for the look direction $u_{L}$ is

$$
\begin{aligned}
y_{L}= & y_{1 L} \cdot y_{2 L}^{*} \\
= & \left|A_{s}\right|^{2} \mathbf{v}_{1 L}^{H} \mathbf{v}_{1 s} \cdot\left(\mathbf{v}_{2 L}^{H} \mathbf{v}_{2 s}\right)^{*} / M N+\left|A_{i}\right|^{2} \mathbf{v}_{1 L}^{H} \mathbf{v}_{1 i} \cdot\left(\mathbf{v}_{2 L}^{H} \mathbf{v}_{2 i}\right)^{*} / M N+ \\
& A_{s} A_{i}^{*} \mathbf{v}_{1 L}^{H} \mathbf{v}_{1 s} \cdot\left(\mathbf{v}_{2 L}^{H} \mathbf{v}_{2 i}\right)^{*} / M N+A_{s}^{*} A_{i} \mathbf{v}_{1 L}^{H} \mathbf{v}_{1 i} \cdot\left(\mathbf{v}_{2 L}^{H} \mathbf{v}_{2 s}\right)^{*} / M N \\
= & \left|A_{s}\right|^{2} B_{1, u_{L}}\left(u_{s}\right) \cdot B_{2, u_{L}}^{*}\left(u_{s}\right)+\left|A_{i}\right|^{2} B_{1, u_{L}}\left(u_{i}\right) \cdot B_{2, u_{L}}^{*}\left(u_{i}\right)+ \\
& A_{s} A_{i}^{*} B_{1, u_{L}}\left(u_{s}\right) \cdot B_{2, u_{L}}^{*}\left(u_{i}\right)+A_{s}^{*} A_{i} B_{1, u_{L}}\left(u_{i}\right) \cdot B_{2, u_{L}}^{*}\left(u_{s}\right),
\end{aligned}
$$

where $B_{1, u_{L}}\left(u_{t}\right)$ is the beam pattern of subarray 1 steered to the direction $u_{L}$ evaluated at $u=u_{t}$ and $B_{2, u_{L}}\left(u_{t}\right)$ is the beam pattern of subarray 2 steered to the direction $u_{L}$ 
evaluated at $u=u_{t}$. The first two terms of (8) correspond to the sources at $u_{s}$ and $u_{i}$, respectively, while the last two terms are crossterms.

Now let us look at detail what happens when we are looking in the direction of the first source, i.e., $\left(u_{L}=u_{s}\right)$ so that what we are trying to get is an accurate estimate of $\left|A_{s}\right|^{2}$. When $u_{L}=u_{s}$, Equation (8) reduces to

$$
\begin{aligned}
y_{L}= & \left|A_{s}\right|^{2}+\left|A_{i}\right|^{2} B_{1, u_{s}}\left(u_{i}\right) \cdot B_{2, u_{s}}^{*}\left(u_{i}\right)+A_{s} A_{i}^{*} B_{2, u_{s}}^{*}\left(u_{i}\right) \\
& +A_{s}^{*} A_{i} B_{1, u_{L}}\left(u_{i}\right) .
\end{aligned}
$$

The desired output in the look direction $u_{L}=u_{s}$ is $\left|A_{s}\right|^{2}$. The second term in (9) $\left|A_{i}\right|^{2} B_{1, u_{s}}\left(u_{i}\right) \cdot B_{2, u_{s}}^{*}\left(u_{i}\right)$ is the standard bias that side lobes always contribute at the look direction in spectral estimation. Assuming that the sources are independent, the product $A_{s} A_{i}^{*}$ or $A_{s}^{*} A_{i}$ will have uniform random phase and averaging snapshots will attenuate the terms containing these products while the term containing $\left|A_{i}\right|^{2}$ will not attenuate with averaging. The term $\left|A_{i}\right|^{2} B_{1, u_{s}}\left(u_{i}\right) \cdot B_{2, u_{s}}^{*}\left(u_{i}\right)$ is the fixed side lobe of the processing independent of the number of observations while the crossterms $\left(A_{s} A_{i}^{*} B_{2, u_{s}}^{*}\left(u_{i}\right)\right.$ and $\left.A_{s}^{*} A_{i} B_{1, u_{L}}\left(u_{i}\right)\right)$ are the terms that attenuate with sufficient observations. The effect of the second term in the total output depends on the power of the second source $\left|A_{i}\right|^{2}$ as well as the side lobe height of the CSA beam pattern steered to $u_{s}$ evaluated at $u_{i}$ which is $B_{1, u_{s}}\left(u_{i}\right) \cdot B_{2, u_{s}}^{*}\left(u_{i}\right)$.
Figure 4 assumes that $\left|A_{i}\right|=10\left|A_{s}\right|$ and the two sources are at $u_{s}=0.45$ and $u_{i}=0$. Both $A_{i}$ and $A_{s}$ are zero mean proper complex Gaussian random variables [15]. The scenario has zero mean white Gaussian noise and the signal-to-noise ratio (SNR) is $0 \mathrm{~dB}$ with respect to the stronger source. The top panel in Figure 4 shows only one snapshot at every look direction while the bottom panel averages over 100 snapshots. As illustrated in the top panel, the effects of the second term $\left|A_{i}\right|^{2} B_{1, u_{s}}\left(u_{i}\right) \cdot B_{2, u_{s}}^{*}\left(u_{i}\right)$ (blue dashed line) and the crossterms $A_{s} A_{i}^{*} B_{2, u_{s}}^{*}\left(u_{i}\right), A_{s}^{*} A_{i} B_{1, u_{L}}\left(u_{i}\right)$ (red dashed-dot line and magenta dashed-dot line) are higher than the desired term $\left|A_{S}\right|^{2}$ (cyan dashed line) at the look direction. Averaging over 100 snapshots reduces the effects of the crossterms as depicted in the bottom panel of Figure 4, but the influence of the second term $\left|A_{i}\right|^{2} B_{1, u_{s}}\left(u_{i}\right) \cdot B_{2, u_{s}}^{*}\left(u_{i}\right)$ (blue dashed line) is still higher than the term $\left|A_{s}\right|^{2}$ (cyan dashed line). Hence, the total power output (black solid line) still has a larger contribution from the second term than the first. In this example, the power spectral estimate at $u_{s}$ is overwhelmed by the interference coming through the side lobe at $u_{i}$, motivating the need for reducing the side lobes of the CSA beamformer.

\section{Analytical expressions for total number of sensors in extended CSA}

This section derives the extension factor required for a CSA to match the peak side lobe level of a full ULA. The

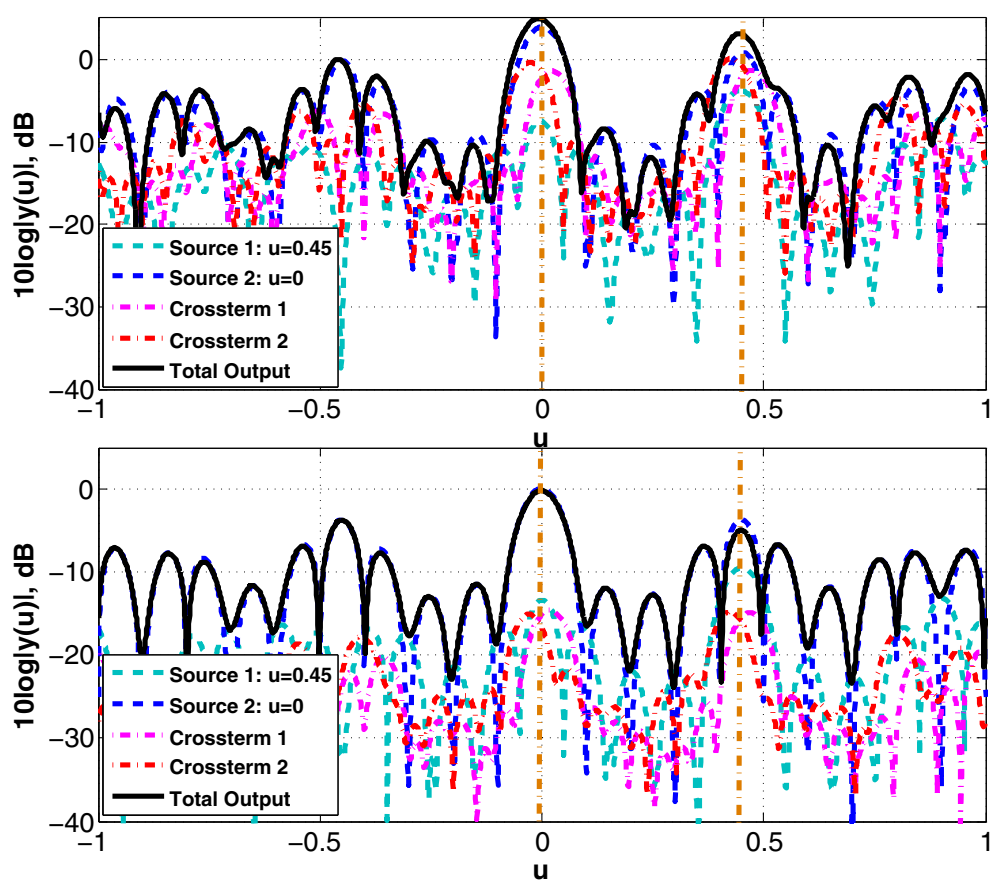

Figure 4 Detection of a weak signal in presence of a strong signal. The top shows high peak side lobes and crossterms in single snapshot case. The bottom shows the reduction of crossterms but no change in peak side lobes with averaging over 100 snapshots. 
extension factor $c$ is the multiplicative factor that each aperture (and number of sensors) must be extended to satisfy the peak side lobe criteria. Assuming that the original CSA has coprime pair $M$ and $N=M+1$, the ECSA will have $c N-1$ and $c N$ sensors in the subarrays. The subarrays share $\lceil c\rceil$ sensors. The total number of sensors in the ECSA is $2 c N-1-\lceil c\rceil$. This section finds the value of $c$ for the uniform, Hann, Hamming, and Dolph-Chebyshev tapers. The expression for $c$ varies among the four tapers studied, but the analysis of uniform, Hann (for $M>4$ ), and Hamming tapers share a common approach since the peak side lobe formation mechanism is similar in these three cases. The common approach follows the following three steps:

1. Without loss of generality, assume that all the beamformers are steered to broadside $u=0$. The peak side lobe for the unextended CSA occurs at the intersection of the adjacent grating lobes of the two subarrays. When $N=M+1$, the intersecting grating lobes will be the first ones at $u=2 / N$ and $u=2 / M$. Because the two grating lobes have equal width for the unextended CSA, this intersection will be exactly at the midpoint at $u=1 / M+1 / N$. Adding sensors to both subarrays while keeping the interelement spacings fixed reduces the side lobe created by the intersection of the first grating lobes. As the subarrays are extended, the beam pattern at the intersection becomes less than the beam pattern at the grating lobe $u=2 / N$. Therefore, the peak side lobe location shifts to be at $u=2 / N$, and the mechanism creating this side lobe is the side lobe of subarray 2 aligned under the grating lobe of subarray 1. The peak side lobe height for the product beam pattern at the location $u=2 / N$ depends on the height of the subarray 2 side lobe that comes under the subarray 1 grating lobe at $u=2 / N$.

2. If the equivalent full ULA beam pattern peak side lobe is psl, then the spatial power spectral estimate from the ULA will have a side lobe of $|\mathrm{psl}|^{2}$. In order for the CSA product power spectral estimate to match this side lobe in the spatial power spectrum, the subarray 2 beam pattern side lobe at $u=2 / N$ must equal $|\mathrm{psl}|^{2}$ since the power spectral estimate will be formed by multiplying this value by the subarray 1 grating lobe with amplitude 1 . The side lobe with amplitude $|\mathrm{psl}|^{2}$ will generally not be the first side lobe of subarray 2 beam pattern but will be more distant. Since beam pattern roll-off factors are generally given in $\mathrm{dB} /$ octave, a $\mathrm{dB}$ scale simplifies the task of finding which subarray 2 sidelobe satisfies the requirement. If the full ULA side lobe in $\mathrm{dB}$ is $\mathrm{SL}=20 \log _{10}|\mathrm{psl}|$, then subarray 2's beam pattern must have a side lobe $2 \mathrm{SL}=20 \log _{10}|\mathrm{psl}|^{2}$. If $R$ is the side lobe roll-off factor, the side lobe with height 2SL $\mathrm{dB}$ is $\mathrm{SL} / R$ octaves away from the peak side lobe location $u_{\mathrm{psl}}$ as shown in the top panel of Figure 5 . Hence, using the expression

$$
u_{p}=u_{\mathrm{psl}} \cdot 2^{\mathrm{SL} / R} \text {, }
$$

gives the $p$ th side lobe location $u_{\mathrm{psl}}$. The peak side lobe location $u_{\mathrm{psl}}$ is the first side lobe location for uniform and Hann taperings and the third side lobe location for Hamming tapering.

3. Find the index $p$ of the side lobe with height $2 \mathrm{SL} \mathrm{dB}$ using the equation $u_{p}=f(p, Q)$, where $f(p, Q)$ is the expression for the $p$ th side lobe location and $f(p, Q)$ is a function of $p$ and the number of sensors in the array $Q$. The $p$ th side lobe is at a distance $f(p, Q)$ in $u$ from its main lobe location in a beam pattern with no grating lobes. The $p$ th side lobe is at a distance $f(p, c N) / M$ in $u$ from a grating lobe location of the subarray 2 beam pattern where the undersampling factor is $M$ and the number of sensors is $c N$ as shown in the bottom panel of Figure 5. Since the first grating lobe of subarray 2 is at $2 / M$, the required $p$ th side lobe is at $2 / M-f(p, c N) / M$. To find the value of $c$ when the subarray 2's $p$ th side lobe aligns with subarray 1's first grating lobe, solve the equation

$$
\frac{2}{N}=\frac{2}{M}-\frac{f(p, c N)}{M}
$$

for $c$.

\subsection{Uniform shading}

As described above, the mechanism for the peak side lobe formation is when the side lobe of one subarray falls under the grating lobe of the other. Use (10) with $R=-6$ $\mathrm{dB} /$ octave [16], $\mathrm{SL}=-13$ and $u_{\mathrm{psl}}=3 / Q$ to find $u_{p}$ for uniform shading. The expression for $p$ th side lobe location is $f(p, Q)=(2 p+1) / Q$ [1]. Solving $u_{p}=f(p, Q)$ for $p$ gives $p=6$. Finally, solving (11) for $c$ yields $c=6.5$. Hence, when the total sensors in the subarrays are $\lceil 6.5 \mathrm{~N}-1\rceil$ and $\lceil 6.5 \mathrm{~N}\rceil$, respectively, the CSA peak side lobe height is less than or equal to the peak side lobe of the equivalent full ULA.

\subsection{Hamming shading}

The treatment of Hamming shading follows the treatment of uniform shading in Section 3.1 very closely. The third side lobe is the peak side lobe with height $-42 \mathrm{~dB}$ and occurs at $u_{\mathrm{psl}}=9 / Q$ [1]. The side lobe roll-off factor is $R=-6 \mathrm{~dB} /$ octave [16]. Equation (10) yields $u_{p}=$ $9 / Q \cdot 2^{42 / 6}$. The $p$ th side lobe for Hamming shading occurs at $u_{p}=(5+2(p-1)) / Q$. Solving $u_{p}=f(p, Q)$ gives $p=575$. When subarray 2's 575th side lobe aligns with the subarray 1's first grating lobe at $u=2 / N$, the peak CSA side lobe is less than or equal to $-42 \mathrm{~dB}$. Solving (11) for 


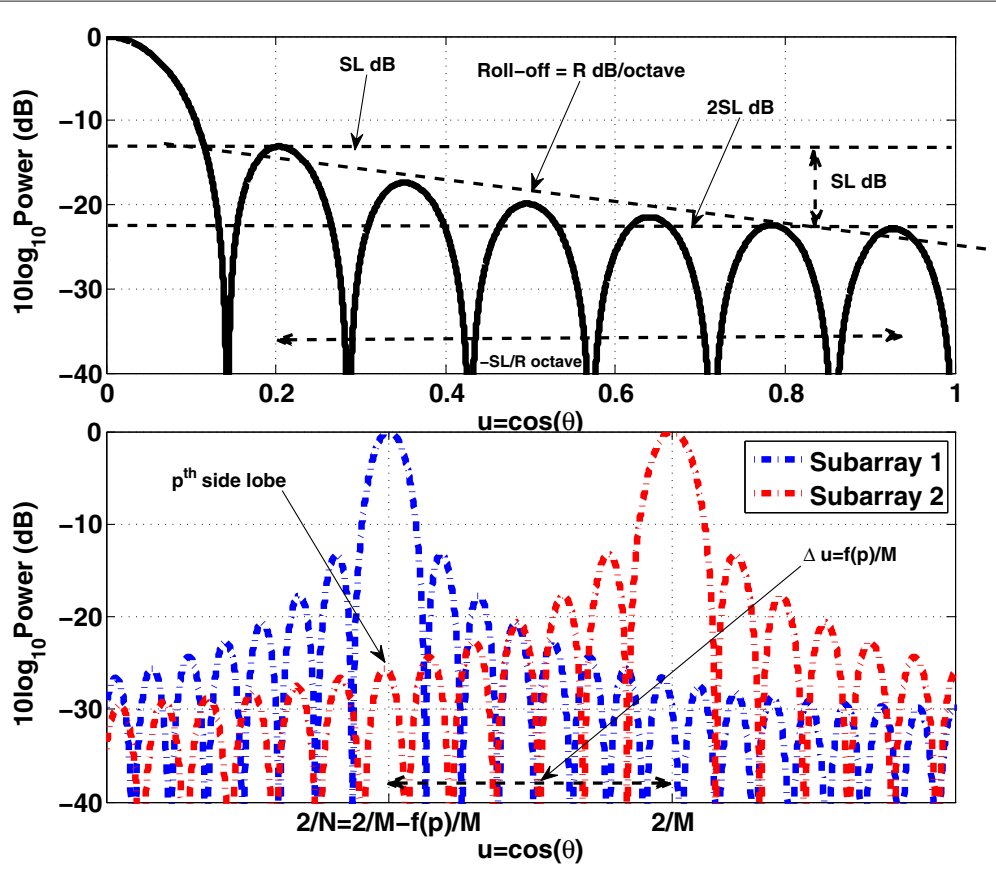

Figure 5 CSA peak side lobe formation mechanism. The top shows the side lobe roll-off factor and the location of the pth side lobe relative to the peak side lobe. The bottom shows the alignment of subarray 2's pth side lobe with subarray 1's grating lobe.

$c$ gives $c=576.5$. The Hamming-shaded ECSA requires $\lceil 576.5 \mathrm{~N}-1\rceil$ and $\lceil 576.5 \mathrm{~N}\rceil$ sensors in the subarrays to match the Hamming-shaded full ULA's peak side lobe. A Hamming-shaded array needs to have at least 1,154 sensors before there is a 575th side lobe. Hence, Hamming shading is not practical for a CSA.

\subsection{Hann shading}

The peak side lobe formation mechanism for Hann tapering is similar to uniform tapering when $M>4$. The $p$ th side lobe of a $Q$ element array occurs at $f(p, Q)=(5+$ $2(p-1)) / Q$, and the side lobe roll-off factor is $R=-18$ $\mathrm{dB} /$ octave [16]. The peak side lobe height is $-32 \mathrm{~dB}$. The side lobe with height $-64 \mathrm{~dB}$ is at $u_{p}=u_{\mathrm{psl}} \cdot 2^{32 / 18}$. Solving the equation $u_{p}=f(p, Q)$ for $p$ gives the index of the side lobe with height $-64 \mathrm{~dB}$ which is 7 . Solving (11) with $p=7$ gives $c=8.5$. The Hann-shaded ECSA with $M>4$ requires $\lceil 8.5 \mathrm{~N}-1\rceil$ and $\lceil 8.5 \mathrm{~N}\rceil$ sensors in the subarrays to match the Hann-shaded full ULA's peak side lobe.

For $M \leq 4$, the peak side lobe for Hann-shaded CSA is caused by the interaction of subarray 1's first side lobe and subarray 2's main lobe rather than a side lobe and a grating lobe. In a Hann-shaded CSA with coprime pair $(2,3)$, when $c$ reaches 5 , the CSA side lobe formed by the interaction of subarray 1's first side lobe and subarray 2's main lobe becomes higher than the side lobe at $u=2 / N$. Increasing $c$ beyond 5 decreases the CSA side lobe at $u=2 / N$ but increases the peak side lobe formed by the main lobe and side lobe interaction. For $c=5$, the peak side lobe level is $-25.3 \mathrm{~dB}$, and this is the minimum possible level. Similarly, for coprime pair $(3,4)$, the minimum peak side lobe is $-28 \mathrm{~dB}$ and the corresponding $c$ is 5.75 . For coprime pair $(4,5)$, the achievable peak side lobe level is $-29.9 \mathrm{~dB}$ and the corresponding value of $c$ is 7 . Hence, the minimum possible side lobe level decreases with increasing $M$, but the peak side lobe height cannot match the equivalent full ULA peak side lobe when $M \leq 5$.

\subsection{Dolph-Chebyshev shading}

The Dolph-Chebychev shading requires a different analysis technique than the shadings discussed above because the Dolph-Chebyshev shading side lobes are equiripple. The Dolph-Chebyshev-shaded subarray beam pattern is given by

$$
B(u)=r \cos \left((P-1) \arccos \left(z \cos \frac{\pi Q u}{2}\right)\right),
$$

where $z=\cos ((\arccos (1 / r)) /(P-1)), r$ is the peak side lobe height, $P$ is the number of sensors and $Q$ is the undersampling factor [17].

Adding sensors to the subarrays reduces the peak side lobe due to the interaction of the first grating lobes at $u=1 / M+1 / N$ as in other shadings. The peak side lobe height at $u=1 / M+1 / N$ becomes less than or equal to the equiripple side lobe level of the subarray after addition of enough number of sensors. At this point, the intersection is no longer creating the peak side lobe but instead the peak side lobe is created by one of the equiripple side lobes 
under a grating lobe. The equiripple nature of DolphChebyshev means the side lobe will not further attenuate with increasing $c$. Increasing $c$ beyond this point may shift the peak side lobe location but not the height since the peak side lobe will be formed by a side lobe of one subarray aligning with one of the grating lobes of the other subarray. Hence, the value of $c$ required to reduce the side lobe at $u=1 / M+1 / N$ to the equiripple side lobe level is the extension factor needed for Dolph-Chebyshev shading. The overall CSA power level will be the same as an individual subarray's amplitude because the CSA power level will be an equiripple side lobe level times a grating lobe level. Achieving the desired side lobe level SL in $\mathrm{dB}$ for the CSA power spectral density requires the individual subarrays to have twice the attenuation 2SL. The value for the extension factor $c$ derived in this paper for Dolph-Chebyshev shaded CSA is the one that matches the Dolph-Chebyshev shaded full ULA with side lobe SL dB using subarrays with $2 \mathrm{SL} \mathrm{dB}$ side lobe. If we apply the same Dolph-Chebyshev parameters to a CSA as a full ULA, the CSA power spectrum peak side lobe will only have half the attenuation of the full ULA equiripple side lobe. Therefore, if we wish to design Dolph-Chebyshev windows for the subarrays to achieve a desired level of attenutation SL, the Dolph-Chebyshev design equation should use 2SL dB to find the shadings for the subarrays.

For an unextended CSA, the peak side lobe is exactly at the intersection of the first grating lobes $u=1 / M+1 / N$ when $N=M+1$ and the contributions of the two subarray beam patterns to the CSA beam pattern at $u=1 / M+$ $1 / N$ are equal. For extended subarrays, the widths of the grating lobes of the two subarrays are slightly different. Hence, the peak side lobe location is only approximately $1 / M+1 / N$. The derivation in this section will assume that the peak side lobe for the ECSA occurs at $1 / M+1 / N$ and also $10 \log _{10}\left(\mid B_{1}(u=1 / M+1 / N, c)\right) \mid \approx 10 \log _{10}\left(\mid B_{2}(u=\right.$ $1 / M+1 / N, c) \mid)$. Finding $c$ to match the peak side lobe SL $\mathrm{dB}$ of the full ULA requires solving the equation

$$
\begin{gathered}
10 \log _{10}\left(\left|B_{1}(u=1 / M+1 / N, c)\right|\right)+10 \log _{10}\left(\mid B_{2}(u=1 / M\right. \\
+1 / N, c) \mid)=\mathrm{SL} .
\end{gathered}
$$

To solve for $c$ more easily, replace (13) with the approximation

$$
10 \log _{10}\left(\left|B_{1}(u=1 / M+1 / N, c)\right|\right)=\mathrm{SL} / 2 .
$$

The equation to be solved in linear scale is $\mid B_{1}(u=$ $1 / M+1 / N, c) \mid=$ psl, where psl $=10^{\mathrm{SL} / 20}$. Evaluating the beam pattern expression (12) for $c M$ with undersampling factor $M+1$ and at the intersection $u=1 / M+1 / N$ and solving the equation resulting from equating the beam pattern magnitude to Dolph-Chebyshev side lobe level $\left(\mathrm{SL}=20 \log _{10}(|\mathrm{psl}|) \mathrm{dB}\right)$ results in

$$
c=\frac{1}{M}+\sqrt{\frac{0.0798 \mathrm{SL}-0.0199 \mathrm{SL}^{2}}{\left.M^{2} \ln \left(\cos \frac{\pi}{2 M}\right)\right)}} .
$$

The Appendix provides the complete derivation of the expression for $c$ given in (15). The Dolph-Chebyshevshaded ECSA requires

$$
c M=1+\sqrt{\frac{0.0798 \mathrm{SL} 0.0199 \mathrm{SL}^{2}}{\left.\ln \left(\cos \frac{\pi}{2 M}\right)\right)}},
$$

and

$$
c M+1=2+\sqrt{\frac{0.0798 \mathrm{SL}-0.0199 \mathrm{SL}^{2}}{\left.\ln \left(\cos \frac{\pi}{2 M}\right)\right)}},
$$

sensor subarrays to match the Dolph-Chebyshev-shaded full ULA's peak side lobe.

\section{Examples}

This section illustrates that extending the CSA subarrays with the number of sensors as derived in Section 3 results in a peak side lobe that matches the full ULA peak side lobe with the same shading.

\subsection{Uniform shading}

For a CSA with coprime pair $(2,3)$ and uniform shading, Section 3.1 predicts that the extended subarrays must include 19 and 20 sensors to match the side lobes of the full ULA. The top panel of Figure 6 plots the extended subarray beam patterns and the CSA product beam pattern. The bottom panel of Figure 6 demonstrates that the ECSA peak side lobe matches the peak side lobe of the equivalent full ULA at $-13 \mathrm{~dB}$.

Figure 7 compares the prediction for the number of sensors required by a uniform-shaded CSA with the number of sensors found through exhaustive simulations to achieve the $-13 \mathrm{~dB}$ side lobe. The dashed line plots the value of $c$ predicted in Section 3.1, while the discrete stars indicate the values found in simulation over a wide range of $M$. The analytical predictions with $c=6.5$ are close to the simulation values.

\subsection{Hann shading}

Figure 8 depicts the beam patterns for ECSAs with Hann shading having coprime pair $(5,6)$. Section 3.3 predicts that the extended subarrays with Hann shading must have 50 and 51 sensors to match the Hann-shaded full ULA peak side lobe. The bottom panel in Figure 8 illustrates that the ECSA peak side lobe is $-32 \mathrm{~dB}$, matching the full ULA peak side lobe height.

Figure 9 compares the prediction for the number of sensors required by a Hann-shaded CSA with the number of sensors found through exhaustive simulations to achieve 

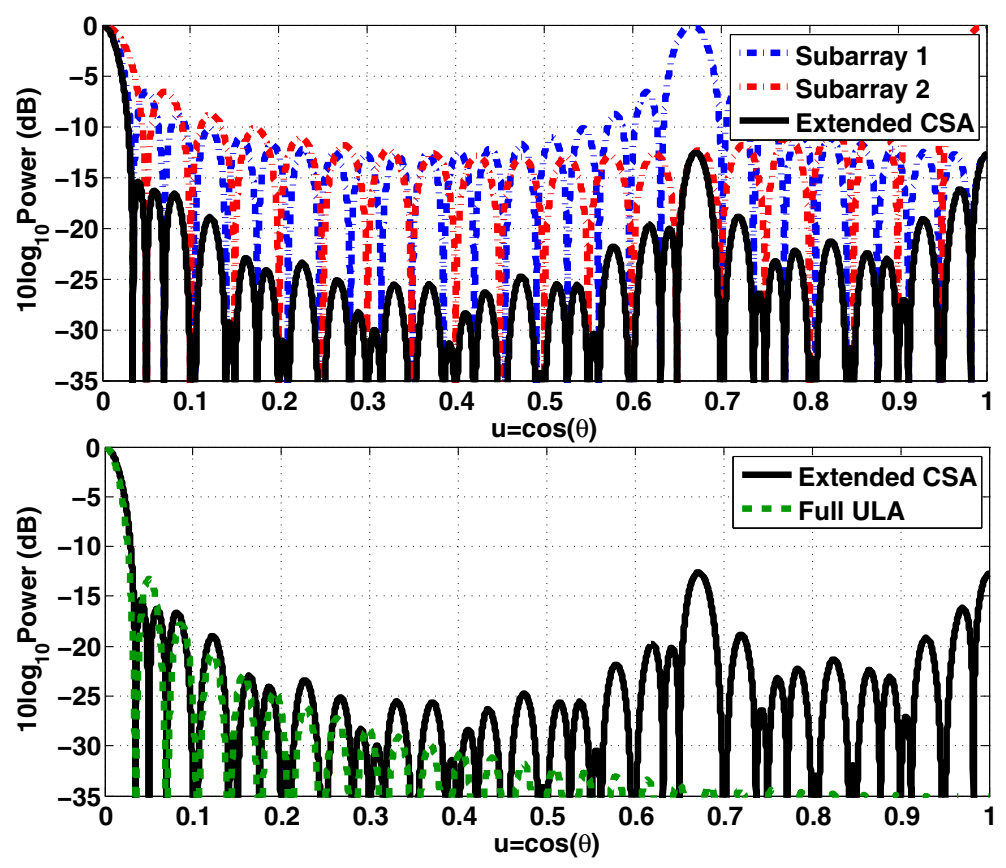

Figure 6 ECSA and equivalent full ULA beam patterns for uniform tapering. The top shows the formation of the beam pattern of an ECSA with coprime pair (2,3), uniform tapering, and 19 and 20 sensors in the extended subarrays. The bottom compares the beam patterns of the ECSA with the beam pattern of the equivalent full ULA.

the $-32 \mathrm{~dB}$ side lobe. The dashed line plots the value of $8.5 \mathrm{M}$ predicted by Section 3.3, while the discrete squares indicate the values found in simulation over a wide range of $M$. The analytical predictions match the simulation values.

\subsection{Dolph-Chebyshev shading}

Substituting SL $=-30 \mathrm{~dB}$ and $M=2$ into (15) yields an extension factor of $c=4.3257$ for a Dolph-Chebyshev shading. The total number of sensors in the subarrays with coprime pair $(2,3)$ have to be at least 9 and 10 . The

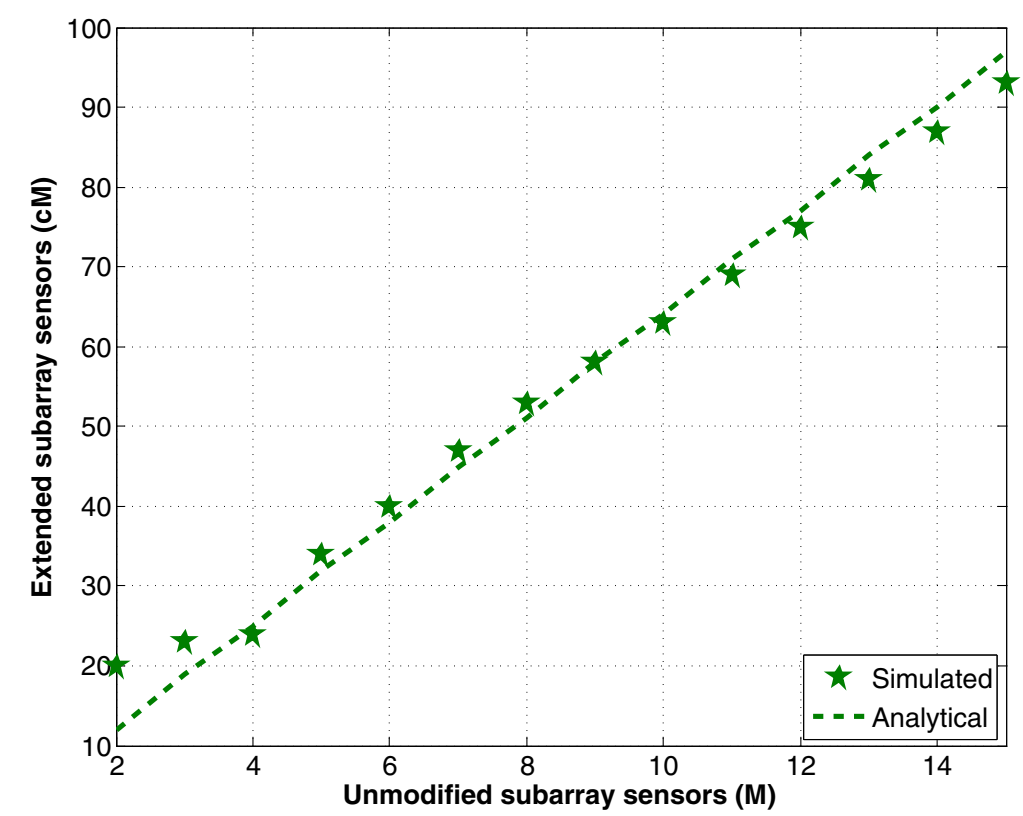

Figure 7 The analytical and empirical values of total sensors in an ECSA with uniform tapering. This figure compares the values for analytical prediction (dashed) and empirical (stars) total sensors in an ECSA with uniform shading. The analytical values agree closely with the empirical values. 

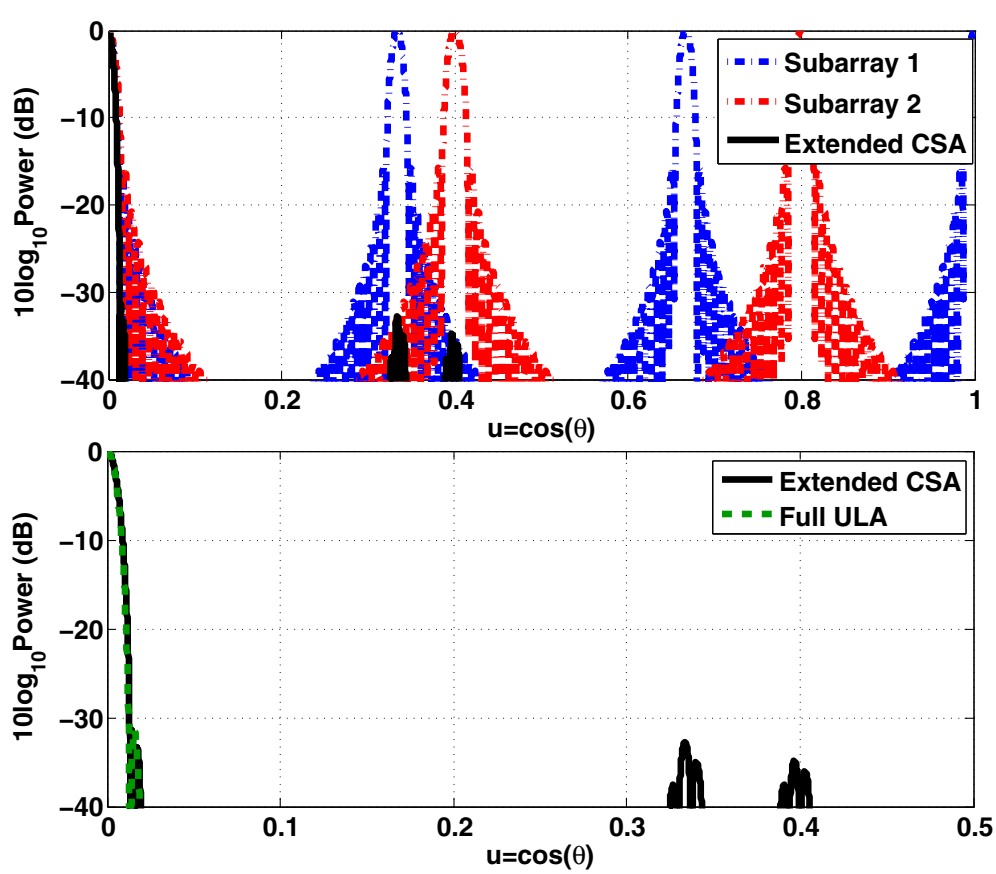

Figure 8 ECSA and full ULA beam patterns for Hann tapering. The top shows the formation of the beam pattern of an ECSA with coprime pair $(5,6)$, Hann tapering, and 50 and 51 sensors in the subarrays. The bottom compares the beam patterns of the ECSA with the beam pattern of the equivalent full ULA.

top panel of Figure 10 shows the beam patterns of the extended subarrays with 9 and 10 sensors and the resulting CSA beam pattern. The bottom panel of Figure 10 compares the CSA beam pattern with the equivalent full ULA beam pattern. The peak side lobes in the CSA and
ULA beam patterns in the bottom panel are equal, confirming the accuracy of (15). Since the subarrays must use Dolph-Chebyshev shading with twice as much side lobe attenuation in $\mathrm{dB}$ as the equivalent full ULA in order for the power spectrum have equal side lobes, the resulting

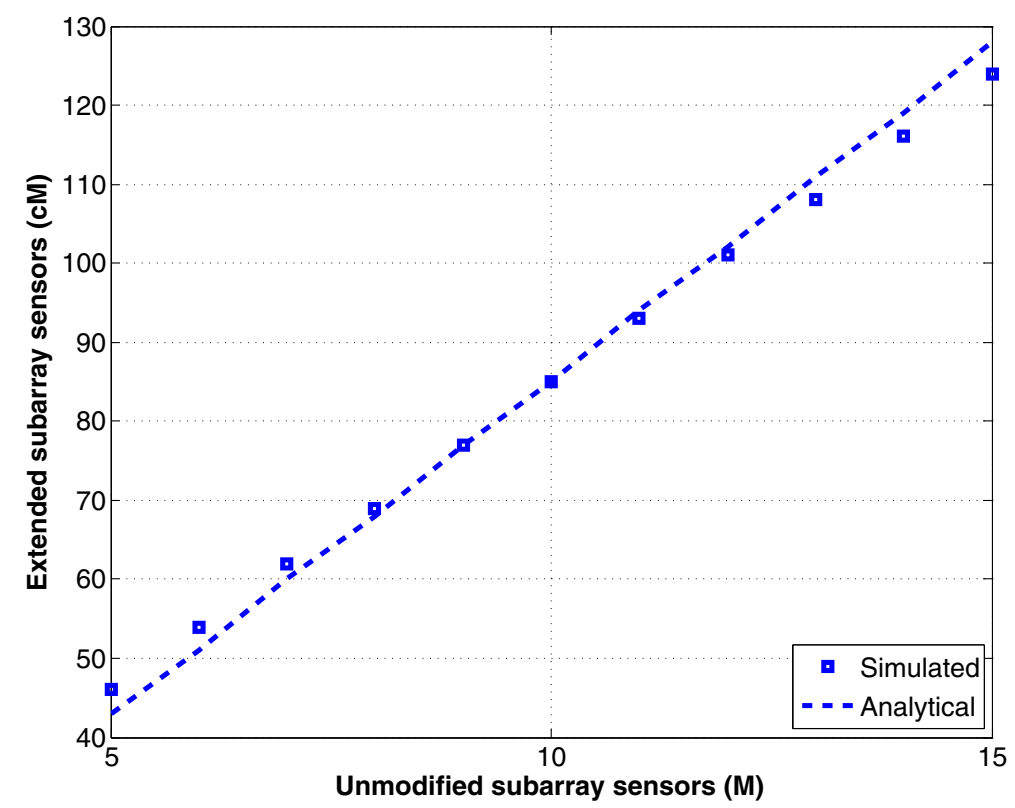

Figure 9 The analytical and empirical values of total sensors in an ECSA with Hann tapering. This figure compares the values for analytical prediction (dashed) and empirical (squares) total sensors in an ECSA with Hann shading. The analytical values agree closely with the empirical values. 

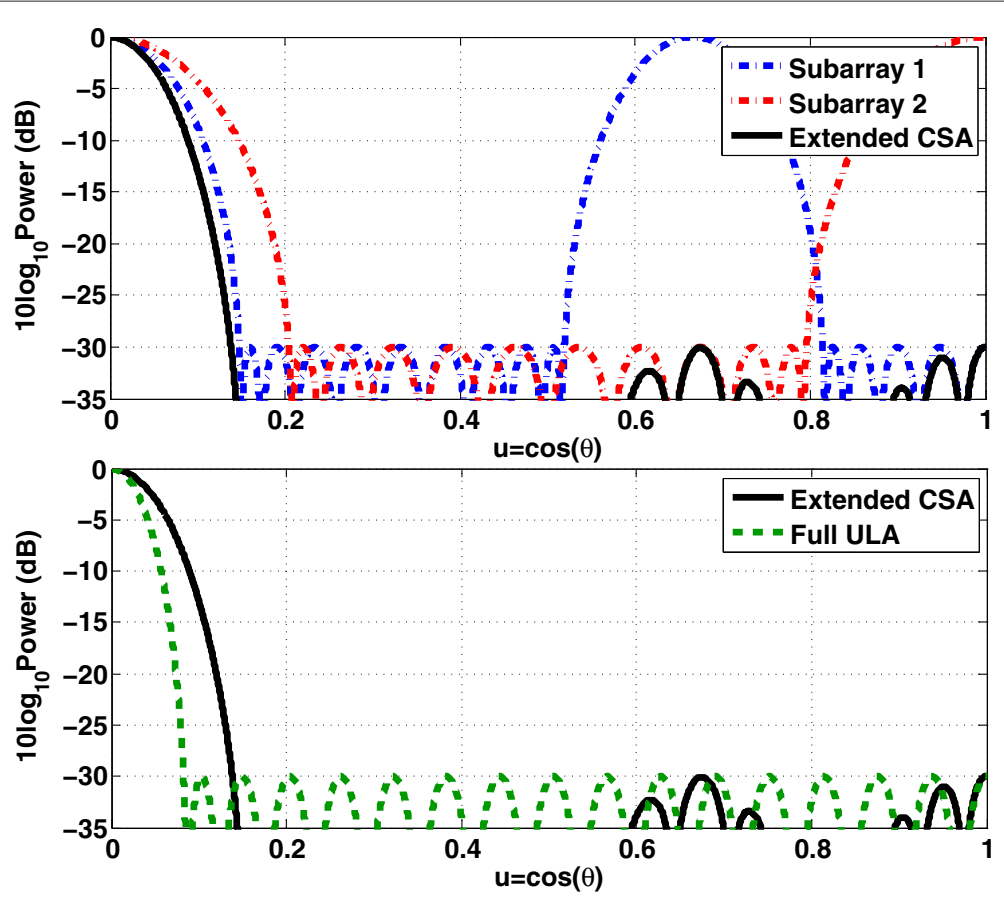

Figure 10 ECSA and full ULA beam patterns for Dolph-Chebyshev tapering. The top shows the formation of the beam pattern of an ECSA with coprime pair (2,3), Dolph-Chebyshev tapering ( $-30 \mathrm{~dB}$ side lobes), and 9 and 10 sensors in the subarrays. The bottom compares the beam patterns of the ECSA with the beam pattern of the equivalent full ULA.

CSA has wider main lobe width and poorer resolution than the ULA.

The value of $c$ for the Dolph-Chebyshev tapering depends on the side lobe level chosen. Decreasing side lobe level increases the main lobe and grating lobe widths resulting in more overlap between the first grating lobes of the two subarrays. Hence, the peak CSA side lobe level for Dolph-Chebyshev shading increases with the decrease in side lobe level of the subarrays requiring more sensors in the ECSA to match the full ULA side lobe level. Figure 11 compares the number of sensors in an extended subarray for Dolph-Chebyshev shading obtained using (16) with the number of sensors obtained from exhaustive simulations for three different side lobe levels, $\mathrm{SL}=-20,-50$, and $-80 \mathrm{~dB}$. The plots confirm that a smaller SL requires more sensors in the ECSA to match the full ULA side lobe level. The dashed lines in the plots indicate the analytical prediction of the number of sensors in the extended subarrays, which agree with the corresponding empirical values indicated by discrete squares.

\section{Discussion}

This section discusses three important CSA design topics. The first topic concerns the coprime pair with minimum peak side lobe for a given aperture. The second topic is a comparison of the extension factor $c$ for different shadings. The third topic is a comparison of the extension scheme described in this paper with the extension scheme proposed by Vaidyanathan and Pal in [2] to achieve a fully populated co-array (ECSA with $2 M$ and $N$ sensors or $M$ and $2 N$ sensors).

\subsection{Coprime pair with minimum peak side lobe for a given aperture}

Consider an application where total aperture is fixed by physical constraints in the problem. To use CSA processing in such array, we need to choose the right coprime pair. Consider an $L$ element ULA with half-wavelength $(\lambda / 2)$ interelement spacing. CSA processing can be applied in a given ULA with several coprime pairs. But with higher coprime pairs, the extension factor $c=6.5$ might not be reached and as a result, the peak side lobe height might be higher than the full ULA peak side lobe $-13 \mathrm{~dB}$. However, the number of sensors used is lower with higher coprime pairs, hence the processing cost is also less. The total number of sensors $T_{s}$ used out of $L$ elements (see Appendix for the complete derivation) is given by

$$
T_{s}=2 \cdot M+1+2 \cdot\left\lfloor\frac{L}{M+1}-M+1\right\rfloor-\left\lceil\frac{\left\lfloor\frac{L}{M+1}+1\right\rfloor}{M}\right\rceil .
$$

For example, for an $L=80$ element ULA, the possible coprime pairs are $(2,3)$ through $(8,9)$. The extension 


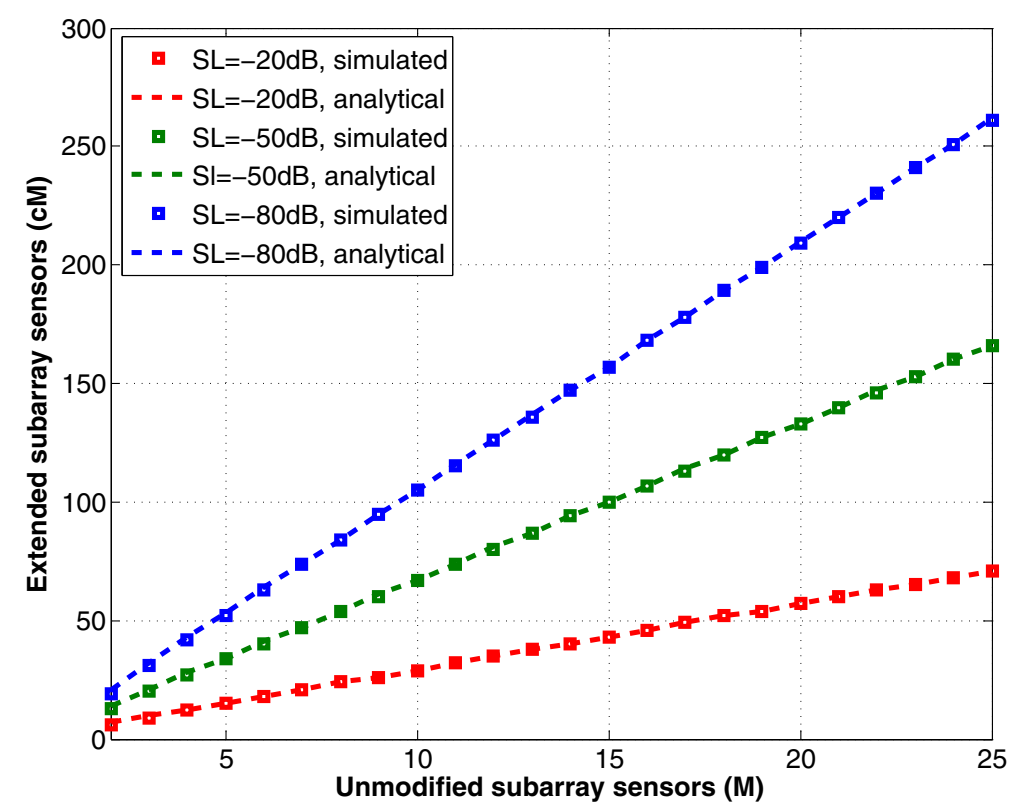

Figure 11 The analytical and empirical values of total sensors in an ECSA with Dolph-Chebyshev tapering. This figure compares the values for analytical and empirical total sensors in an ECSA with Dolph-Chebyshev shading at different side lobe levels. Different colors represent different side lobe levels. The analytical values comply with the empirical values for all the side lobe levels examined.

factor $c$ is highest with coprime pair $(2,3)$ and lowest with $(8,9)$. The coprime pair $(2,3)$ is the only pair that allows $c$ to be as high as 6.5 which is the required value for uniform tapering. Hence, the peak side lobe is below $-13 \mathrm{~dB}$ only in case of the coprime pair $(2,3)$. For all other pairs, the peak side lobe is higher than $-13 \mathrm{~dB}$ as depicted in
Figure 12. The number of sensors required to span the original 80 element aperture is greatest for the case of $(2,3)$ which is the least sparse of the coprime pairs. The number of sensors is fewest for the case of $(8,9)$, which is the most sparse of the available coprime pairs, as shown in Figure 13.

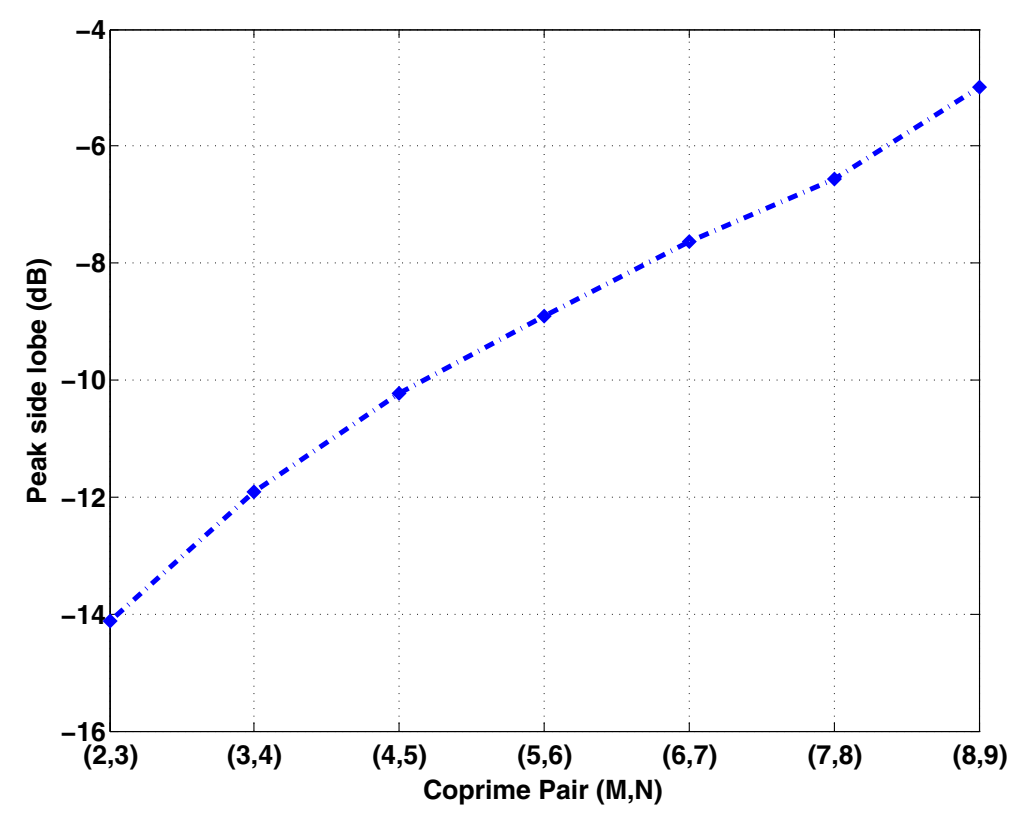

Figure 12 Comparison of peak side lobes in uniform-shaded CSAs with different coprime pairs for a fixed aperture. This figure compares peak side lobes in CSAs with different coprime pairs where the aperture is fixed at $80 \lambda / 2$. Higher coprime pairs exhibit higher peak side lobes. 


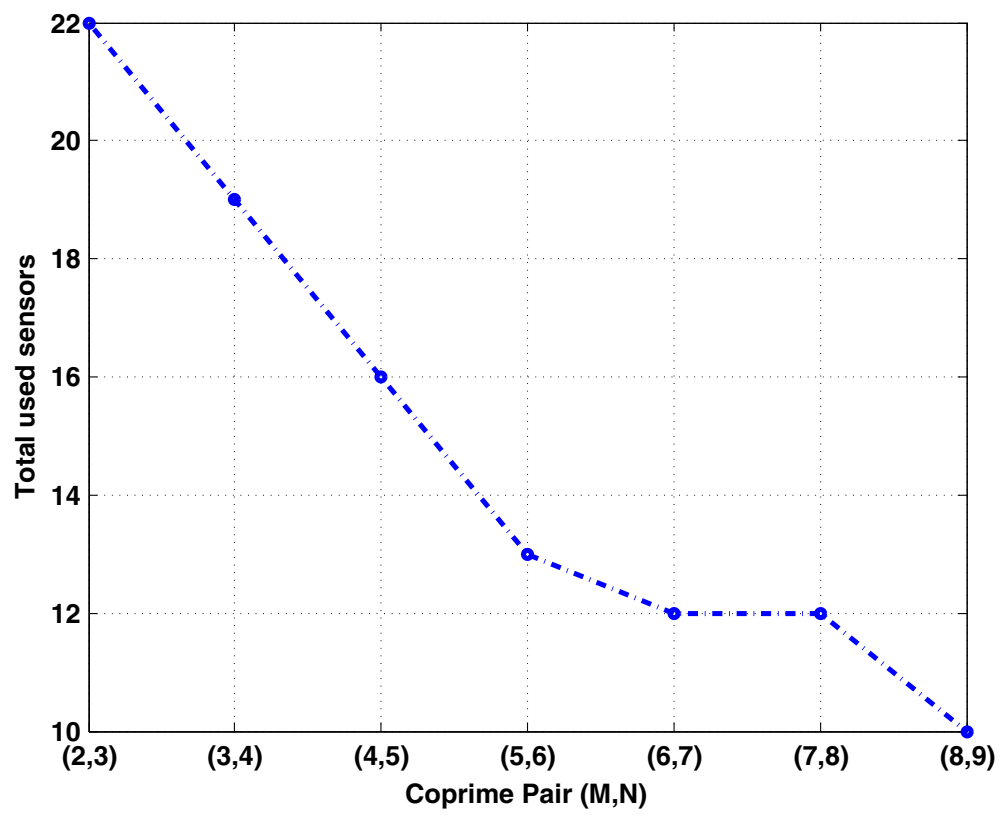

Figure 13 Comparison of the number of used sensors in CSAs with different coprime pairs for a fixed aperture. This figure compares the total number of sensors in CSAs with different coprime pairs where the aperture is fixed at $80 \lambda / 2$. Higher coprime pairs have fewer total number of sensors.

\subsection{Comparison of the extension factor $c$ for different shadings}

Section 3 predicts different values of $c$ for different shadings. Comparison of the values of $c$ for different shadings provides insight into peak CSA side lobe formation and reduction mechanism. In general, the windows with narrower main lobes for a given number of sensors and larger side lobe roll-off factor require fewer total sensors in the ECSA. This section compares the total number of sensors in an ECSA with the different shadings studied in Section 3.

Figure 14 illustrates the number of sensors in an extended subarray for different coprime pairs $(M, M+1)$ for different shadings. Among uniform (green dash-dot line with circles) and Hann (blue dashed line with circles) shadings, uniform shading requires the fewest number of sensors to guarantee that the peak side lobe is less than the full ULA peak side lobe. Uniform shading is then followed by Hann and Hamming shadings in that order as expected. Since the number of sensors in an ECSA with Dolph-Chebyshev shading depends on the side lobe attenuation level, we can compare the Dolph-Chebyshev shading with any other shading by requiring that DolphChebyshev shading match the side lobe attenuation of the other shading. The value of $c$ for Dolph-Chebyshev shading with side lobe levels equal to other shadings $(-13 \mathrm{~dB}$ for uniform, $-32 \mathrm{~dB}$ for Hann, and $-42 \mathrm{~dB}$ for Hamming) are summarized in Table 1 . The data from Table 1 predict that Dolph-Chebyshev shading requires fewer sensors than the uniform, Hann, or Hamming shadings to provide the same peak side lobe levels. Figure 14 confirms that Dolph-Chebyshev shading with $-13 \mathrm{~dB}$ side lobe (green dash-dot line with squares) requires fewer sensors than the uniform-shaded CSA, and Dolph-Chebyshev shading with $-32 \mathrm{~dB}$ side lobe (blue dashed line with squares) requires fewer sensors than the Hann-shaded CSA. Achieving CSA spectral estimates with peak side lobes of $-13,-32$, and $-42 \mathrm{~dB}$ requires that the CSA subarrays have Dolph-Chebyshev shadings with side lobe levels $-26,-64$, and $-84 \mathrm{~dB}$, respectively. Subarray beam patterns with such low side lobe levels have wide main lobes. The Dolph-Chebyshev shaded CSAs with -13 or $-32 \mathrm{~dB}$ peak side lobes have wider main lobe widths than corresponding uniform and Hann shaded CSAs as corroborated by Figure 15. The Dolph-Chebyshev shaded CSAs that match the uniform and Hann shaded CSAs peak side lobes cannot match their respective resolution. One advantage, however, of using Dolph-Chebyshev shading is smaller aperture than the corresponding uniform or Hann shadings as shown in Figure 16. The choice of a shading for a CSA is a trade-off among side lobes, resolution, and aperture.

\subsection{Comparison of the extension schemes}

Vaidyanathan and $\mathrm{Pal}$ [2] proposed extending CSAs by extending only one of the subarrays, resulting in subarrays of $2 M$ and $N$ sensors or $2 N$ and $M$ sensors depending on which subarray is extended. Their motivation for this 


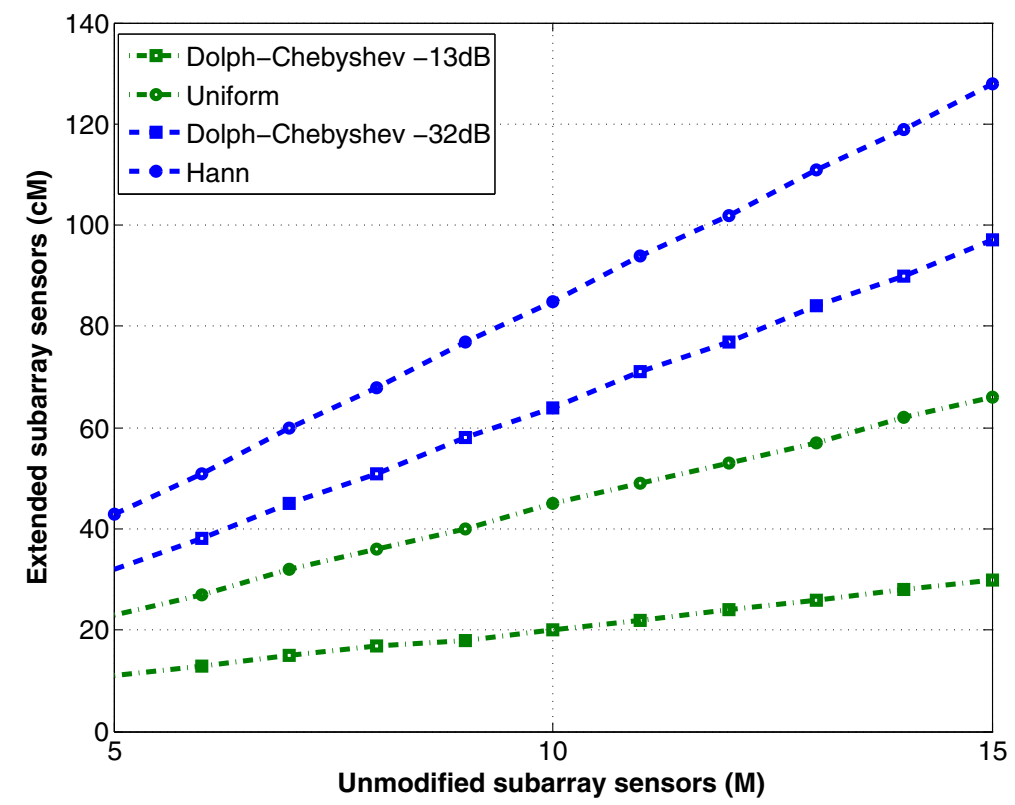

Figure 14 Comparison of the total sensors in a subarray for different taperings. This figure compares the total sensors in a subarray of the ECSA required for Dolph-Chebyshev (squares) vs. uniform and Hann windows (circles) over a range of aperture. Different colors and line types represent different side lobe levels. For any array size examined, Dolph-Chebyshev window requires fewer sensors to achieve the side lobe level than the fixed windows.

array extension scheme is to achieve a difference co-array that fully populated all of the lags. It is an interesting question to contrast the single array extension scheme proposed by Vaidyanathan and Pal (referred to as the SAE for the remainder of this discussion) with the ECSA approach proposed in this paper which extends both subarrays. Three parameters that characterize the CSAs are the peak side lobe, the number of sensors, and the aperture. Any meaningful comparison will hold at least one of these parameters equal while comparing the other two. The following paragraph compares the two CSA extension schemes for each of the three cases, constraining one of the parameters to be equal while assessing the other two parameters. In each case, the ECSA is chosen to match the peak side lobe of the equivalent resolution full ULA as presented in Section 3. For each comparison, the SAE is designed to have the same peak side lobe, number of sensors, or aperture as appropriate for that comparison.

Table 1 Comparison of the values of $c$ for Dolph-Chebyshev shading with uniform, Hann, and Hamming shadings

\begin{tabular}{lcc}
\hline $\begin{array}{c}\text { Peak side } \\
\text { lobe level }\end{array}$ & $\begin{array}{c}\boldsymbol{c} \text { for } \\
\text { Dolph-Chebyshev }\end{array}$ & $\begin{array}{c}\boldsymbol{c} \text { for } \\
\text { other shading }\end{array}$ \\
\hline$-13 \mathrm{~dB}$ & 2 & 6.5 (Uniform) \\
$-32 \mathrm{~dB}$ & 4.4 & 8.5 (Hann) \\
$-40 \mathrm{~dB}$ & 11 & 576.5 (Hamming) \\
\hline
\end{tabular}

Constraining the ECSA and SAE to have the same peak side lobe leads to an SAE with slightly more sensors than the ECSA for uniform, Hann, and Dolph-Chebyshev shading as shown in Figure 17. For uniform shading, ECSA has smaller aperture for $M<6$ and for Hann shading, ECSA has smaller aperture for $M<8$. For Dolph-Chebyshev also, ECSA has smaller aperture for smaller $M$ and the exact value of $M$ when the SAE begins to have smaller aperture depends on the equiripple side lobe level. Constraining the ECSA and SAE to have equal number of sensors for uniform, Hann, or Dolph-Chebyshev shading results in an SAE with higher peak side lobe. The ECSA aperture is smaller than SAE for smaller $M$. Constraining the ECSA and SAE to have equal aperture results in the SAE with higher peak side lobe and about $M$ fewer sensors than ECSA. In all the three cases discussed above, the difference in resolution is negligible and both ECSA and SAE yield a filled difference coarray in the range 0 to $M N$. In conclusion, ECSA performs better than SAE.

\section{Conclusions}

This paper derived analytical expressions for the total numbers of sensors required in ECSAs to guarantee that the peak side lobe is not greater than the peak side lobe of the full ULA with the same aperture. The analytical expressions were derived for several standard shadings viz, uniform, Hann, Hamming, and Dolph-Chebyshev. 


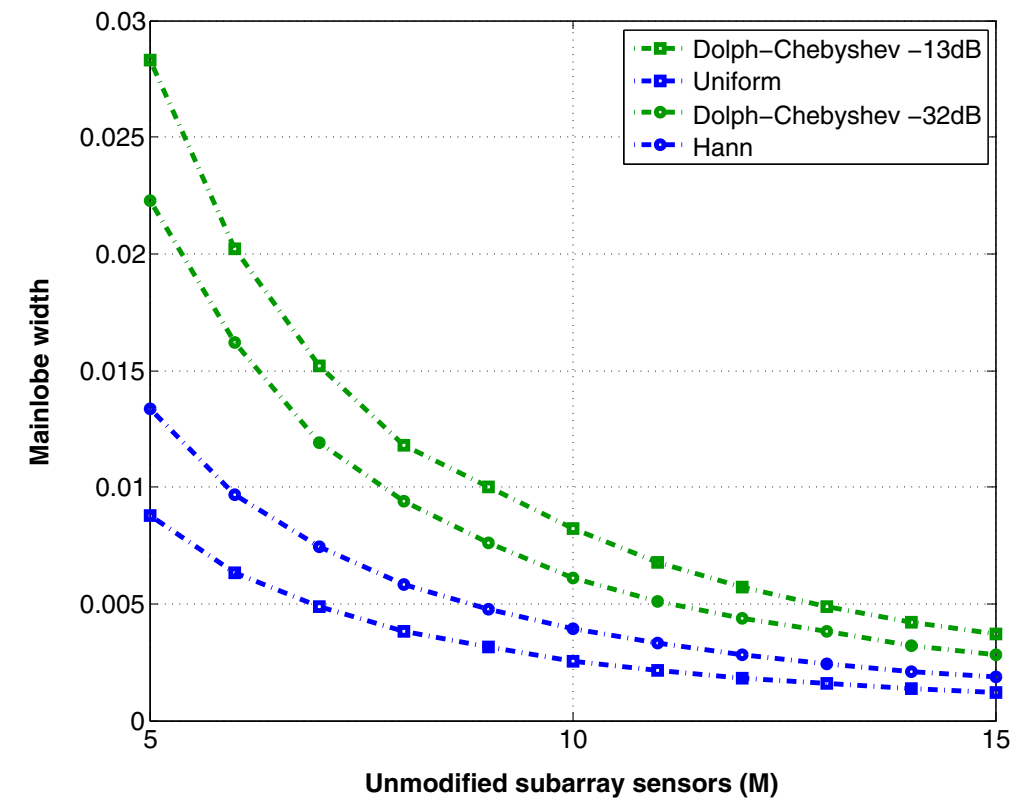

Figure 15 Comparison of the main lobe width in an ECSA for different taperings. This figure compares the main lobe width in an ECSA for Dolph-Chebyshev (squares) vs. fixed type windows (circles) over a range of aperture. Different colors and line types represent different side lobe levels. For any array size examined, Dolph-Chebyshev window has wider main lobe width than the fixed windows.

The comparison of the numbers of sensors in different shadings showed that for a given coprime pair, uniform shading requires fewer sensors than Hann and Hamming shadings. Though Dolph-Chebyshev requires fewer sensors than uniform, Hann, and Hamming shadings to match the respective side lobes, Dolph-Chebyshev shading has less resolution than the corresponding uniform, Hann, or Hamming shadings. The best shading for a situation depends on whether side lobe height or number of sensors is the primary consideration. The comparison

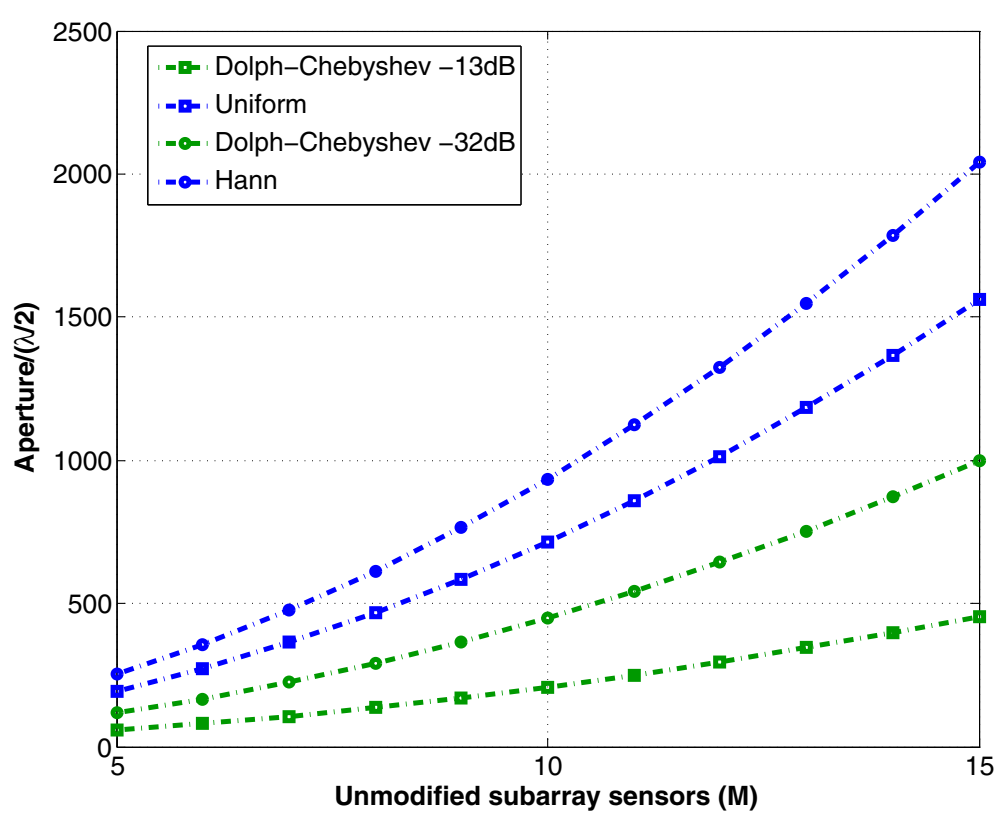

Figure 16 Comparison of the ECSA aperture for different taperings. This figure compares the ECSA aperture of Dolph-Chebyshev (squares) vs. fixed type windows (circles) over a range of aperture. Different colors and line types represent different side lobe levels. For any array size examined, Dolph-Chebyshev window has smaller aperture than the fixed windows. 


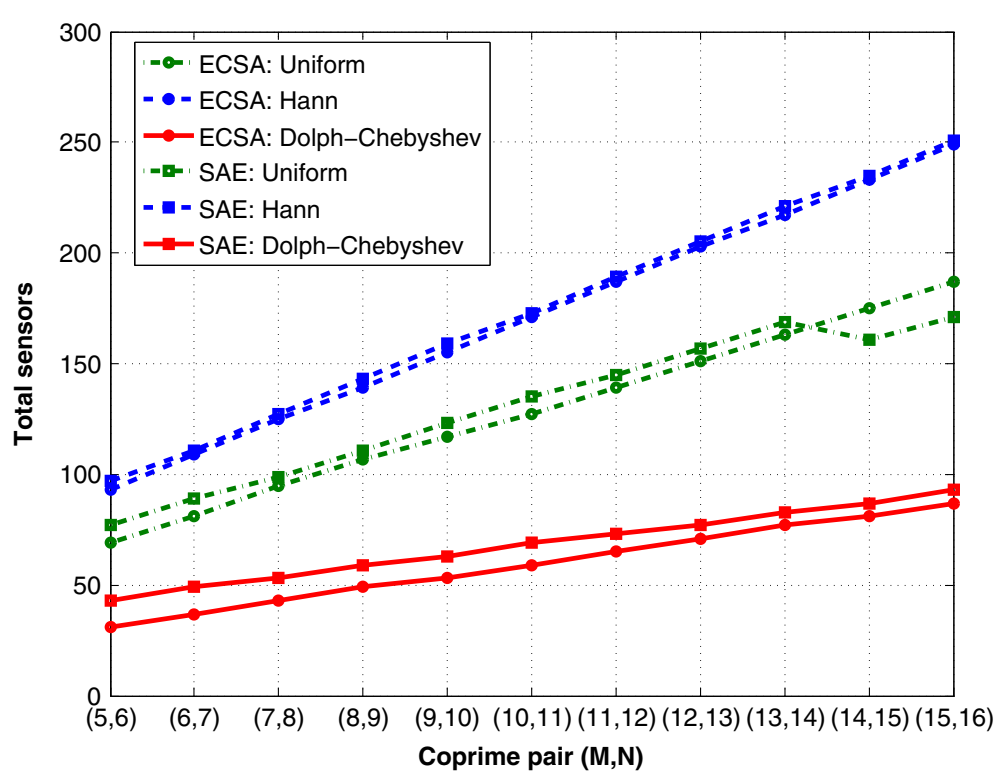

Figure 17 Comparison of the total sensors in ECSA and SAE for different shadings. This figure compares the ECSA and SAE total sensors to match the peak side lobe of the ECSA's equivalent full ULA. Different colors and line types represent different shadings, and the different symbols represent different extension schemes. For any array size and shading examined, ECSA requires fewer sensors to match the equivalent full ULA's peak side lobe.

of the two different extension schemes showed that ECSA performs better than SAE.

\section{Appendix}

\section{Extension factor for Dolph-Chebyshev shading}

The extended subarrays have the total sensors $c M$ and $c M+1$ and undersampling factors $M+1$ and $M$, respectively. Substituting these values into (12) yields

$$
\begin{gathered}
B_{1}(u, c)=r \cos \left((c M-1) \arccos \left(z_{1} \cos \left(\frac{\pi(M+1) u)}{2}\right)\right)\right), \\
B_{2}(u, c)=r \cos \left(c M \arccos \left(z_{2} \cos \left(\frac{\pi M u)}{2}\right)\right)\right),
\end{gathered}
$$

where $z_{1}=\cos ((\arccos (1 / r)) /(c M-1))$ and $z_{2}=$ $\cos ((\arccos (1 / r)) /(c M))$. Since $r<<1, \arccos (1 / r) \approx$ $j \ln (2 / r)$ (see Appendix). Hence, $z_{1}$ and $z_{2}$ can be approximated as

$$
z_{1} \approx \cos (j \ln (2 / r) /(c M-1))
$$

and

$$
z_{2} \approx \cos (j \ln (2 / r) /(c M)) .
$$

The peak CSA side lobe occurs approximately at $u=$ $1 / M+1 / N$, where the desired product of the magnitudes of the subarray beam patterns equals the Dolph-
Chebyshev side lobe level $r$. Thus, the magnitude of $B_{1}(u=1 / M+1 / N, c)$ is approximately $\sqrt{r}$ which leads to the following equation

$$
\left|\cos \left((c M-1) \arccos \left(-z_{1} \cos \frac{\pi}{2 M}\right)\right)\right|=\frac{1}{\sqrt{r}} .
$$

Since $-z_{1} \cos (\pi /(2 M))<-1$ for all practical values of $r, c$ and $M$ and $\arccos (x)=\pi-\arccos (-x)$ for $x<-1$, the LHS of (21) becomes

$$
\left|\cos \left((c M-1) \pi-(c M-1) \arccos \left(z_{1} \cos \frac{\pi}{2 M}\right)\right)\right|=\frac{1}{\sqrt{r}}
$$

which simplifies to

$$
\cos \left((c M-1) \arccos \left(z_{1} \cos \frac{\pi}{2 M}\right)\right)=\frac{1}{\sqrt{r}} .
$$

Because the value of $\alpha=(c M-1) \arccos \left(z_{1} \cos \right.$ $(\pi / 2 M))$ is purely imaginary with magnitude greater than 1 , the approximation $\cos \alpha \approx \exp (-j \alpha) / 2$ simplifies (23) to

$$
\cosh \left(\frac{\ln (2 / r)}{c M-1}\right) \cos \left(\frac{\pi}{2 M}\right)=\cosh \left(\frac{\ln \left(\frac{2}{\sqrt{r}}\right)}{c M-1}\right)
$$

Taking natural $\log$ of the both sides of (24) and using a first order Taylor series expansion for $\ln \left(\left(4^{A}+r^{2 A}\right) /\right.$ 
$\left.\left(4^{A}+r^{A}\right)\right)$ about $A=0$, where $A=1 /(c M-1)$ finally yields

$$
c=\frac{1}{M}+\sqrt{\frac{0.0798 \mathrm{SL}-0.0199 \mathrm{SL}^{2}}{\left.M^{2} \ln \left(\cos \frac{\pi}{2 M}\right)\right)}} .
$$

\section{Proof of $\arccos (1 / r) \approx j \ln (2 / r)$ for small $r$}

Consider the equation $\arccos (1 / r)=f$. Choosing $r<0.1$ as a practical range of side lobe levels results in a purely imaginary $f$ with magnitude exceeding 3. Exploiting Euler's relation yields $(\exp (j f)+\exp (-j f)) / 2=1 / r$. The large imaginary $f$ insures that the $\exp (j f)$ term is negligible, and some algebra yields $f \approx j \ln (2 / r)$ for $r \ll 1$.

\section{Total sensors in a CSA of aperture $L \lambda / 2$}

Since the ECSA aperture has to be equal to the available aperture,

$$
\left(M+a_{\text {sensors }}-1\right)(M+1) \lambda / 2=L \lambda / 2,
$$

where $a_{\text {sensors }}$ is the additional sensors in each subarray. Solving (26) for $a_{\text {sensors }}$ gives

$$
a_{\text {sensors }}=\left\lfloor\frac{L}{M+1}-M+1\right\rfloor .
$$

Since in each $M$ sensors of subarray 1 , one sensor is shared with subarray 2, the total shared sensors in an ECSA is $\lceil c\rceil$ which is given by $\lceil c\rceil=\left\lceil\left(M+a_{\text {sensors }}\right) / M\right\rceil$. Plugging in the expression for $a_{\text {sensors }}$ from (27) expands the total shared sensors to

$$
\lceil c\rceil=\left\lceil\frac{\left\lfloor\frac{L}{M+1}+1\right\rfloor}{M}\right\rceil .
$$

The total sensors in an ECSA is $T_{s}=M+a_{\text {sensors }}+N+$ $a_{\text {sensors }}-\lceil c\rceil$ which gives us

$$
T_{s}=2 \cdot M+1+2 \cdot\left\lfloor\frac{L}{M+1}-M+1\right\rfloor-\left\lceil\frac{\left\lfloor\frac{L}{M+1}+1\right\rfloor}{M}\right\rceil .
$$

\section{Competing interests}

The authors declare that they have no competing interests.

\section{Authors' contributions}

KA performed this research as part of her PhD dissertation under the guidance of JRB and KEW. All authors read and approved the final manuscript.

\section{Acknowledgements}

Kaushallya Adhikari and John Buck were supported by the Office of Naval Research Basic Research Challenge Program grant N00014-13-1-0230. Kathleen Wage was supported by the Office of Naval Research Basic Research Challenge Program through award N00014-13-1-0229.

\section{Author details}

${ }^{1}$ Electrical and Computer Engineering Department, University of Massachusetts Dartmouth, 285 Old Westport Road, North Dartmouth, MA 02747, USA. ${ }^{2}$ Electrical and Computer Engineering Department, George Mason University, 4400 University Dr, Fairfax, VA 22030, USA.
Received: 27 May 2014 Accepted: 10 September 2014 Published: 27 September 2014

\section{References}

1. HVan Trees, Optimum Array Processing: Detection, Estimation and Modulation Theory, Part IV). (John Wiley and Sons, Inc., New York, 2002)

2. PP Vaidyanathan, $P$ Pal, Sparse sensing with co-prime samplers and arrays. IEEE Trans. Signal Process. 59(2), 573-586 (2011). doi:10.1109/TSP.2010.2089682

3. PP Vaidyanathan, P Pal, Theory of sparse coprime sensing in multiple dimensions. IEEE Trans. Signal Process. 59(8), 3592-3608 (2011). doi:10.1109/TSP.2011.2135348

4. DD King, RF Packard, RK Thomas, Unequally-spaced, broad-band antenna arrays. IRE Trans. Antennas Propagation AP-8, 380-385 (1960) doi:10.1109/TAP.1960.1144876

5. H Unz, Linear arrays with arbitrarily distributed elements. IRE Trans. Antennas Propagation 8(2), 222-223 (1960). doi:10.1109/TAP.1960.1144829

6. R Harrington, Sidelobe reduction by nonuniform element spacing. IRE Trans. Antennas Propagation 9(2), 187-192 (1961). doi:10.1109/TAP.1961.1144961

7. AL Maffett, Array factors with nonuniform spacing parameter. IRE Trans. Antennas Propagation 10(2), 131-136 (1962) doi:10.1109/TAP.1962.113783

8. A Ishimaru, Theory of unequally-spaced arrays. IRE Trans. Antennas Propagation 10(6), 691-702 (1962). doi:10.1109/TAP.1962.1137952

9. A Moffet, Minimum-redundancy linear arrays. IEEE Trans. Antennas Propagation 16(2), 172-175 (1968). doi:10.1109/TAP.1968.1139138

10. X Zhu, JR Buck, Designing nonuniform linear arrays to maximize mutual information for bearing estimation. The J. Acoust. Soc. Am. 128(5), 2926-2939 (2010). doi:10.1121/1.3488665

11. SR Tuladhar, JR Buck, Optimum array design to maximize fisher information for bearing estimation. The J. Acoust. Soc. Am. 130(5), 2797-2806 (2011). doi:10.1121/1.3644914

12. P Pal, PP Vaidyanathan, Nested arrays: a novel approach to array processing with enhanced degrees of freedom. IEEE Trans. Signal Process. 58(8), 4167-4181 (2010). doi:10.1109/TSP.2010.2049264

13. P Pal, PP Vaidyanathan, Nested arrays in two dimensions, part I: geometrical considerations. IEEE Trans. Signal Process. 60(9), 4694-4705 (2012). doi:10.1109/TSP.2012.2203814

14. K Adhikari, JR Buck, KE Wage, Beamforming with extended co-prime sensor arrays, in 2013 IEEE International Conference on Acoustics, Speech and Signal Processing (ICASSP) (IEEE, Vancouver, 2013), pp. 4183-4186. doi:10.1109/ICASSP.2013.6638447

15. PJ Schreier, LL Scharf, Statistical Signal Processing of Complex-valued Data: The Theory of Improper and Noncircular Signals. (Cambridge University Press, New York, 2010)

16. FJ Harris, On the use of windows for harmonic analysis with the Discrete Fourier Transform. IEEE Proc. 66(1), 51-83 (1978). doi:10.1109/PROC.1978.10837

17. CL Dolph, A current distribution for broadside arrays which optimizes the relationship between beam width and side-lobe level. IRE Proc. 34(6), 335-348 (1946). doi:10.1109/JRPROC.1946.225956

doi:10.1186/1687-6180-2014-148

Cite this article as: Adhikari et al:: Extending coprime sensor arrays to achieve the peak side lobe height of a full uniform linear array. EURASIP Journal on Advances in Signal Processing 2014 2014:148. 